\title{
Assimilation of DEMs generated from optical stereo and InSAR pair through data fusion
}

\author{
Ashutosh Bhardwaj ${ }^{1}$, Rajat Subhra Chatterjee ${ }^{1}$, Kamal Jain ${ }^{2}$ \\ ${ }^{1}$ Indian Institute of Remote Sensing (ISRO), Dehradun, India \\ ${ }^{2}$ Indian Institute of Technology, Roorkee, India
}

\section{Email address:}

ashutosh@iirs.gov.in(A. Bhardwaj), rschatterjee@iirs.gov.in(R. S. Chatterjee), kjain_us@yahoo.com(K.Jain)

\section{To cite this article:}

Ashutosh Bhardwaj, Rajat Subhra Chatterjee, Kamal Jain. Assimilation of DEMs Generated from Optical Stereo and InSAR Pair through Data Fusion. Science Research Vol. 1, No. 3, 2013, pp. 39-44. doi: 10.11648/j.sr.20130103.12

\begin{abstract}
Main objective of this study is to compare and assimilate the digital elevation models (DEMs) generated using C-band Envisat, L-band ALOS PALSAR Interferometric SAR (InSAR) datasets and Cartosat-1 optical stereo data. The study area is Dehradun city and surroundings including lower Shivalik hills i.e. the younger Himalayas in the south and higher Himalayas on the north. The general elevation of the area ranges from 400m to 2000m AMSL. Ground control points (GCPs) collected from Differential Global Positioning System (DGPS) survey, were used for satellite triangulation of optical data \& geocoding of InSAR data. RMSE for satellite triangulation for cartosat-1 is 0.745 using 6 ground control points and 3 check points. It is found that when GCP's are used along with reference DEM (SRTM), the DSM generated by ALOS PALSAR and DSM generated from Cartosat-1 stereo data are well representing the terrain. The fusion of imagery from multiple sensors has been in use in remote sensing for scientific exploration in the past but there is a little work done on data fusion application for generation of topography (DEM) through Optical stereo and SAR interferometry data sources The defined framework for data fusion uses three fundamental steps in the analysis of the system namely, identification, estimation, and validation for algorithm preparation. The knowledge of the study area, i.e. the field, and the processes involved in the DEM generation of optical and InSAR data are cognitively used for rule-based decision making in the algorithm for mathematical generation of resultant DEM through data fusion implemented at feature level.
\end{abstract}

Keywords: Satellite Photogrammetry, SAR Interferometry, Data Fusion, DGPS, DEM

\section{Introduction}

Data fusion is a process dealing with the association, correlation, and combination of data and information from single and multiple sources to achieve refined position and identity estimates, and complete timely assessments of situations and threats as well as their significance [1]. Algorithm engineering is a combination of theoretical algorithm design with real-world data. The primary goal of algorithm design by theoreticians is efficiency. Implementation of a data fusion system is a complex task. There is no perfect algorithm that is optimal under all conditions. Several of the most critical issues related to the implementation are requirements analysis, sensor selection, architecture selection, algorithm selection, software implementation and testing and evaluation [2]. However, with the increased computer power and development of new and novel detection systems, the data produced needs to be handled in a robust and logical manner. As such computer systems have been developed that are capable of extracting meaningful information from the recorded data. The integration of data, recorded from a multiple sensor system, together with knowledge, is known as data fusion [3]. Data (or, more generically, information) fusion may proceed at different levels, like the level of dynamics, the level of attributes, and the level of evidence [4]. Fig. 1 shows the System based data fusion architecture.

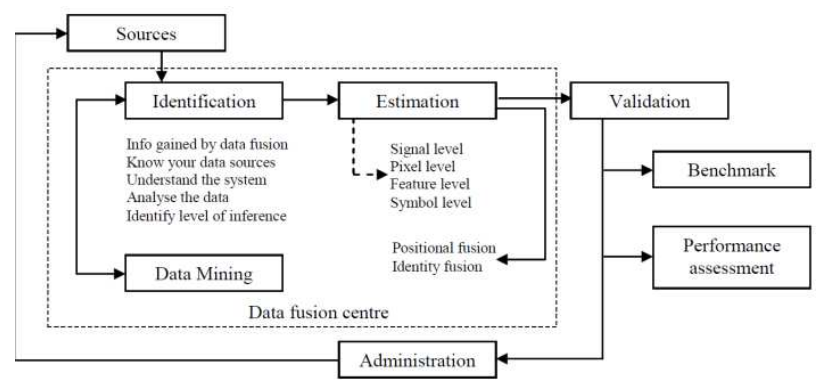

Figure 1. System based data fusion architecture [3] 
Today data is easily available from High Resolution (HR) to Very High Resolution (VHR) optical and microwave sensors to suffice data for mapping and modelling applications. The issue of generation of a good DSM and DEM are critical to the representation of surface as well as topography for different applications. The elevation (ground or surface) is mathematically derived through various approaches such as through the traditional use of surveying equipments (e.g. traverses using theodolite or levels), aerial/satellite photogrammetry, LiDAR, or SAR interferometry (InSAR/IfSAR) [5]. Digital terrain models and orthoimages were generated for planetary missions, such as to represent the topography of Mars and Moon using, Mars Express (MEX) High-Resolution Stereo Camera (HRSC) [6] and Chandrayaan-1 using, Terrain Mapping Camera (TMC) [7] respectively besides laser altimeters (MOLA/LOLA). Attempts are being made by investigators for generating DEMs using fusion. DEM is generated for a part of Antarctica by fusing multi-temporal, multi-sensor and multi-source elevation data to address the ice elevation change to infer the ice mass balance [8].

Primarily, the HR optical payloads have been IKONOS, Cartosat-1/2/2A/2B, QuickBird, Worldview-1/2, Geoeye1/2, Pléiades-HR 1A/1B. Grodecki and Dial, has shown through their various studies the utility of RPCs [9]. Many studies on Cartosat-1 data have shown the good accuracy and utility of cartosat-1 stereo data for mapping [10] [11] [12] and fusion. To generate error free DEM from Cartosat-1 stereo imagery in high mountainous regions there is a requirement of post processing techniques like fill-gap solutions, filtering process or manual editing. Stereo triplet from Cartosat-1 was used to generate DEM for mountainous topography. This DEM shows better reconstruction of elevation model even at occluded region when compared with simple stereo pair based DEM. Planimetric and height accuracy (RMSE) of nearly $3 \mathrm{~m}$ were obtained and qualitative analysis shows reduction of outliers at occluded region [13].

Microwave remote sensing is able to overcome the two main limitations or requirements of optical data i.e. its dependency on sun illumination and day time operation and so gaining importance particularly for disaster management. SRTM, Radarsat-1/2, ERS-1/2, TerraSAR-X, TanDEM-X, ALOS-PALSAR, COSMO Skymed-1/2/3/4 has proven the SAR applications for various applications including DEM generation. Interferometric SAR is a technique to derive $3 \mathrm{D}$ or elevation information of terrain using the phase information implicit in the return signals. It utilizes the phase difference between corresponding pixels of two SAR images of the same area but acquired from two different locations in the orbit(s). The resulting interferogram is a contour map of the change in distance between the ground and the radar instrument. Some of the factors which limits the interferometric measurements are surface preservation, decorrelations, phase ambiguity, platform limitations calling for a precise navigation system,
Cycle-slicing limit, and others [14]. In SAR, Interferometric pair can be achieved by: a) single or simultaneous pass interferometry, or b) Repeat or dual pass interferometry. The selection parameters for InSAR include: view angle (ascending and descending pass), spatial or geometrical baseline, temporal baseline, time of acquisition, meteorological conditions, coherence. Two waves with a constant phase difference over a period of time are called coherent waves. The measured backscatter will depend on imaging geometry, reflectivity (i.e. dielectric constant) and surface roughness. In microwave remote sensing, mainly the Fraunhoffer criterion is used to define a surface smoothness besides the Rayleigh's criteria. The undulations should be less than $\mathrm{h}<(\lambda / 32 \cos \theta)$, where $\theta$ is the incidence angle so that a surface can be called as smooth. A surface like, road, calm water, dry soil will have dominating coherent scattering phenomenon depending on the incident wavelength. A rough surface will result in noncoherent behaviour. Repeat pass-interferometry cannot be used for water features as water decorrelates very fast. As a general rule it can be assumed that the coherence decreases with increasing master-slave acquisition time distance [15]. Gorokhovich et al. has shown in their results that absolute average vertical errors from CGIAR dataset can range from $7.58 \pm 0.60 \mathrm{~m}$ to $4.07 \pm 0.47 \mathrm{~m}$ (mean \pm S.E.M.), which is significantly better than a standard SRTM accuracy value (i.e. $16 \mathrm{~m}$ ) [16]. Studies have shown that the vertical accuracy of an ASTER DEM approaches $25 \mathrm{~m}$, but this can improve to $9-11 \mathrm{~m}$ in areas with less vegetation cover [17]. The horizontal and vertical errors usually cannot be separated: incorrect elevation could appear at the correct location, or the error may be due to a correct elevation in the wrong location [18]. This emphasizes on the significance of correct geocoding using the ground control points (GCPs) [19]. The potential of fusing several DEMs generated using different sensors (SPOT-5 optical stereo images, radarsat-2 stereo images, COSMO-SkyMed InSAR image pairs) and methods to provide a gapless DEM with improved overall accuracy is shown for Hengduan mountain, China [20].

The aim of the presented research work is to model and evaluate the automatically generated digital elevation model (DEM) through multisensory data fusion system.

\section{Study Area}

The study area comprises of Dehradun and its surrounding areas in Uttarakhand. Fig. 2, depicts the undulating nature of the study area. The area predominantly comprises of hills and valleys. In the south, it has agricultural plain area and has a large forest cover with lot of seasonal streams. The general elevation of the area ranges from $400 \mathrm{~m}$ to $2000 \mathrm{~m}$ AMSL. 


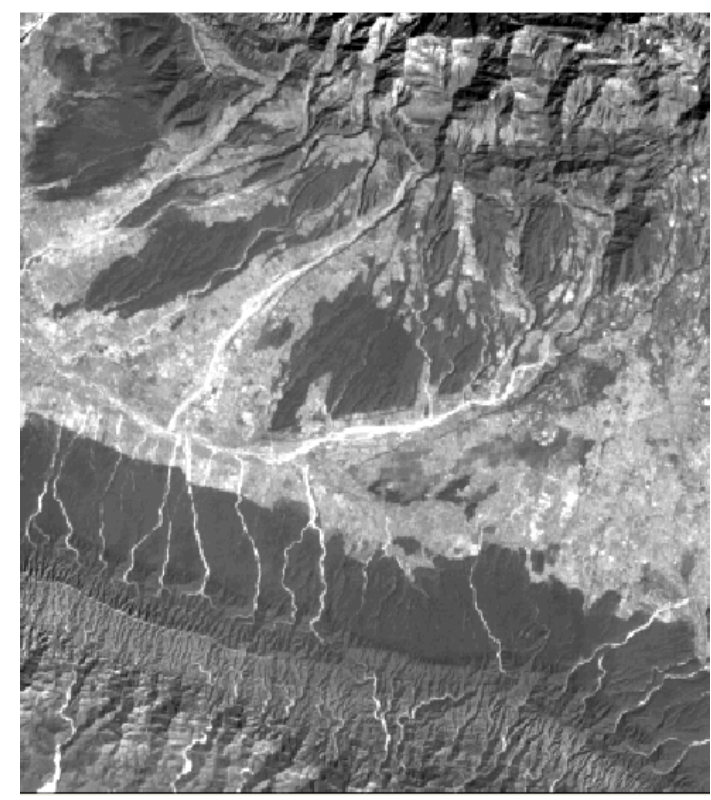

Figure 2. Dehradun \& surroundings (Cartosat-1 aft image)

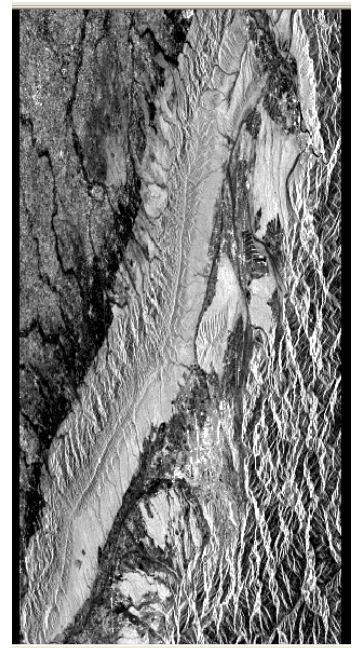

Fig 3(a). Master Image

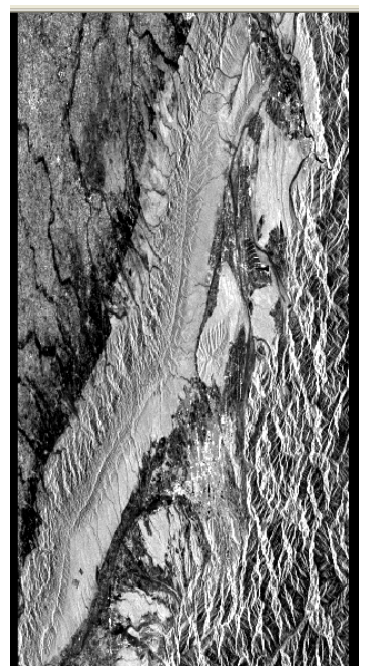

Fig 3(b). Slave Image

\section{Data Used}

Following datasets were used in this study:

a) Optical data: Table 1, gives the details of Cartosat-1 stereopair used here. Indian Remote Sensing Satellite Cartosat-1 is designed to cater to applications in cartography, terrain modeling, large-scale mapping, and carries two PAN sensors for stereo capability with $2.5 \mathrm{~m}$ spatial resolution to acquire two images simultaneously, one forward looking at +26 degrees and other one is aft at 5 degrees for near instantaneous stereo data.

Table 1. Cartosat-1 scene details

\begin{tabular}{cc}
\hline & Cartosat-1 Scene Details \\
\hline Center Lat & $30.35141408 \mathrm{~N}$ \\
Center Lon & $77.91245328 \mathrm{E}$ \\
Satellite ID & P5 \\
Sensor & PAF \\
Path-Row & $0526-258$ \\
Date & 05 FEB 2006 \\
Orbit Number & 4091 \\
Number of Bands & 1 Pan \\
Radiometric Resolution & $10 \mathrm{bits}$ \\
\hline
\end{tabular}

b) Microwave data: ALOS PALSAR (Phased Array type L-band Synthetic Aperture Radar) is an active microwave sensor using L-band frequency to achieve cloud-free and day-and-night land observation. The master image and slave image are shown in Fig. 3(a, b). InSAR pair summary for the scenes of Dehradun \& surroundings is given below in Table 2. Envisat InSAR data (HHPolarization) is also used for DEM generation and analysis. Since the coherence in the Envisat InSAR pair is poor, it is not discussed here further.

Table 2. Some parameters for the ALOS PALSAR data

\begin{tabular}{ccc}
\hline Parameters & Master Image & Slave Image \\
\hline Scene_ID & ALPSRP207230590 & ALPSRP213940590 \\
Orbit_Precision & Precision & Precision \\
Attitude_Precision & Standard & Standard \\
Sc_Center_Time & $2009121417: 21: 04.010$ & $2010012917: 20: 56.653$ \\
Sc_StartTime & $2009121417: 20: 59.743$ & $2010012917: 20: 52.386$ \\
Sc_End_Time & $2009121417: 21: 08.277$ & $2010012917: 21: 00.920$ \\
OffNadirAngle & 34.3 & 34.3 \\
ProductDataSize & 1334.1 & 1334.1 \\
NoOfPixels & 9344 & 9344 \\
NoOfLines & 18432 & 18432 \\
\hline
\end{tabular}

c) SRTM data as reference DEM for InSAR pair.

d) Ground control points collected through a differential GPS (DGPS) survey.

\section{Methodology}

The methodology for generating fused DEM by incorporating a rule-based approach utilizing the optical stereo data and InSAR pair is given in Fig. 3. The data fusion at feature level is implemented here. The optical stereo data is processed for satellite triangulation and DEM generation in LPS software. Sarscape software is used for 
generation of DEM from InSAR pairs (ALOS-PALSAR and Envisat) using ground control points (GCPs) and reference DEM (SRTM). Subsets for the common area were prepared from the ALOS PALSAR DEM and Cartosat-1 DEM at $10 \mathrm{~m}$ resolution. The vector file was generated using the GCPs. The InSAR pair is found to have reasonable coherence, considering the topography and the vegetation cover in the study area. The forest cover is seen as bright in the ALOS-PALSAR image due to high moisture content and thus high dielectric constant as compared to neighbouring pixels. Baseline estimation was carried out to generate the baseline and orbital parameters for the InSAR pair. These are useful in assessing the feasibility and quality of InSAR processing. Both the DEMs (10m resolution) from Cartosat-1 stereo and ALOS PALSAR InSAR pair were than compared and then fused together at feature level using a model based on rule-based approach. The comparison of the resulting DEM was carried out with the individual DEMs. In the present study, it was hypothesized that the fused DEM generated using the developed model will improve the DEM.

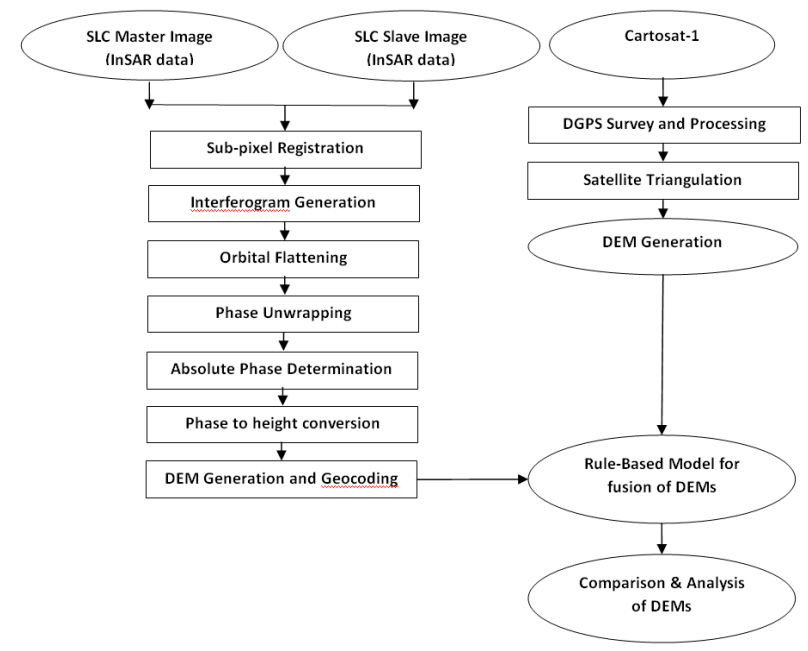

Figure 3. Methodology for DEM generation and analysis

\section{Procedure, Analysis and Results}

The Digital Elevation Models (DEMs) were generated using InSAR processing and satellite photogrammetry for ALOS-PALSAR InSAR pair (Fig. 4) and Cartosat-1 stereo datasets (Fig. 5), respectively. DEM generated using Cartosat-1 was validated using the Hold-Out-Validation (also known as the test sample estimation) available in the LPS software. The fused DEM is shown in Fig. 6. It is seen that there is a large difference in elevation at few point's due to temporal decorrelation, as ALOS PALSAR (master/slave image) has revisit period of 46 days and small difference at points to as much as $1.03 \mathrm{~m}$. Master image was acquired on 14.12.2009 at 17:21:04.010" and slave image is acquired on 29.01.2010 at 17:20:56.653", attributing to the geometrical, temporal and volume scattering decorrelation. For comparison between the
DEMs a region of interest (ROI) over a part of the overlap area was generated for an area of 157.215 square $\mathrm{km}$ comprising 2,51,544 pixels and the difference in elevation in ALOS PALSAR DEM and Cartosat-1 DEM was calculated at each pixel within the ROI. It resulted in difference image with mean of $-23.18 \mathrm{~m}$ and standard error of $1.12 \mathrm{~m}$. The large differences at places are attributed to the hilly terrain, dense vegetation. The histogram is shown in Fig. 7.

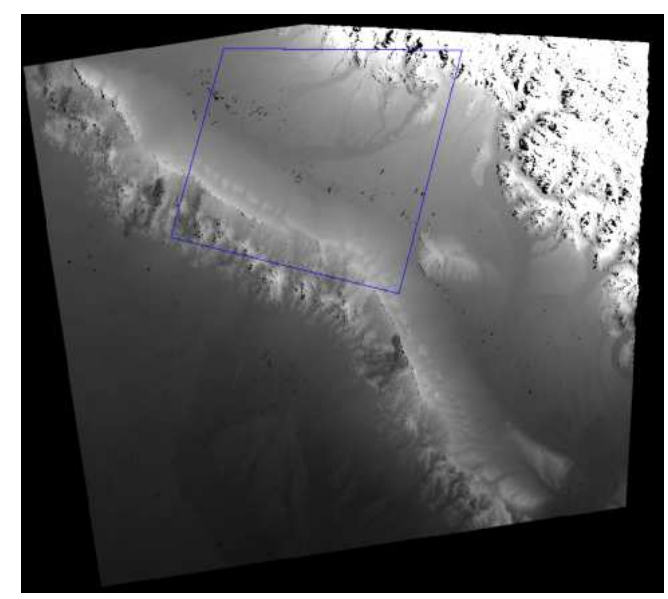

Figure 4. DEM generated using ALOS PALSAR InSAR data

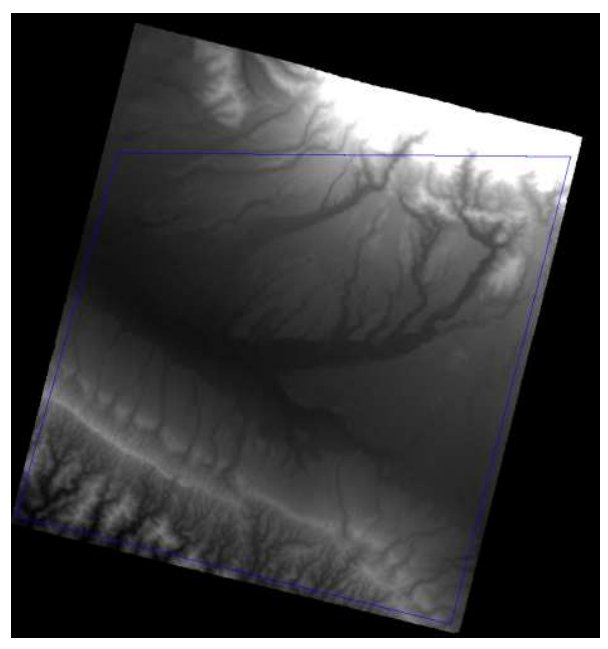

Figure 5. DEM generated from Cartosat-1 stereopair

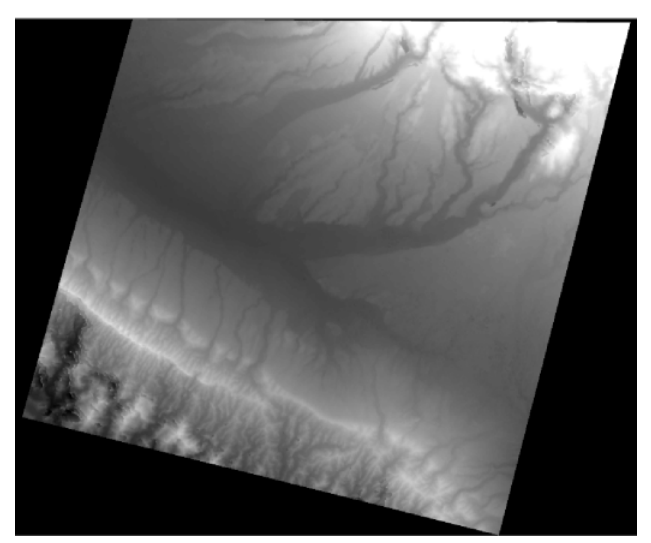

Figure 6. DEM prepared from data fusion 


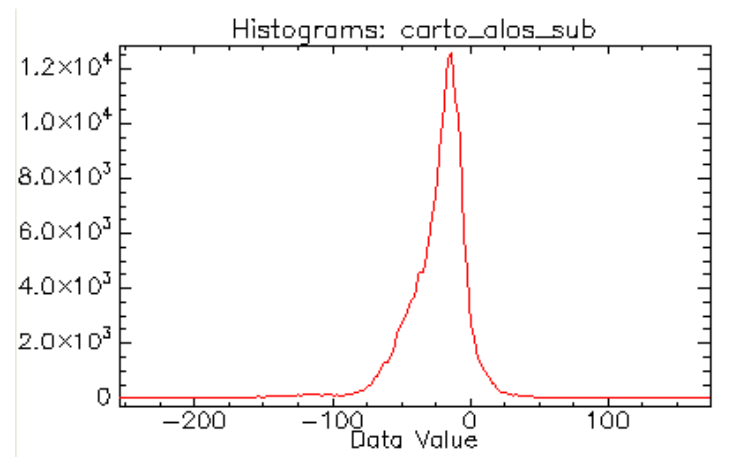

Figure 7. Histogram for the difference image

The polygon (blue colour) in Fig. 4 and Fig. 5 shows the common area between the DEMs generated from ALOS PALSAR and Cartosat-1. The main outputs generated in the process (SARScape) were normal baseline $(736.9 \mathrm{~m})$, Range shift (76.491), Azimuth shift (61.204), interferogram and coherence images. Fig. 8 shows a zoomed view of the interferogam showing the interferogram fringes. Then the interferogram flattening is used to remove the phase differences caused due to difference in the position of terrain with respect to the two antennae positions. The constant phase (due to the acquisition geometry) and the phase expected for a flat Earth or for a known topography (SRTM here) are separated from the residual differential phase. The DEM flattening is executed by transforming the input DEM into the master slant range image geometry using precise orbital information and accurately geocoded reference DEM.

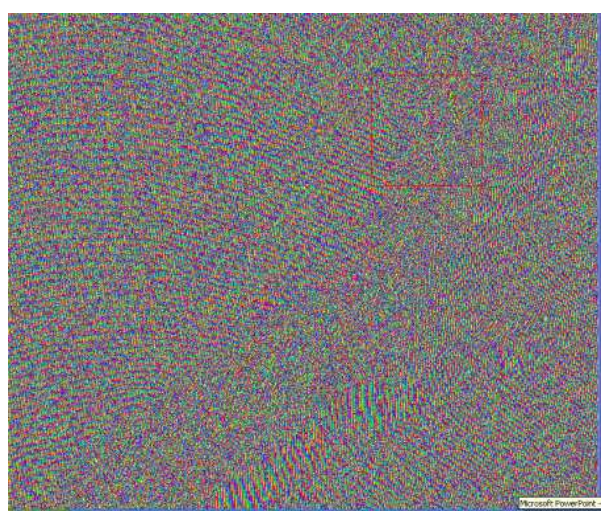

Figure 8. Interferogram

The flattened interferogram is filtered to give an improved phase signal to noise $(\mathrm{S} / \mathrm{N})$ ratio. Here, from the master and slave coregistered complex SAR images, interferometric correlation or coherence $(\gamma)$ is calculated as a ratio between coherent and incoherent summations. The Interferometric Coherence, which is an indicator of the phase quality, is also generated. Phase Unwrapping is automatically done to resolve the $2 \pi$ ambiguity. As seen from the coherence image (Fig. 9), it can be seen that the coherence is reasonably good in this InSAR pair.

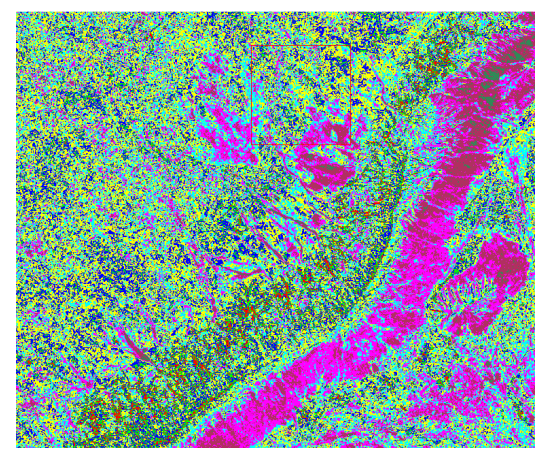

Figure 9. Coherence Image

The orbital parameters are corrected using vector files having $8 \mathrm{GCPs}$, in the orbital refinement process. The orbit correction parameters are written in the header file of the input unwrapped phase. This step is crucial for a correct transformation of the phase information into height (or displacement) values. This Orbital refinement is used as an input for the height generation process. Terrain height is generated from the interferometric unwrapped phase and geocoding is completed in UTM (43 zone North). Pixels with coherence values smaller than this threshold are set to dummy $(\mathrm{NaN})$ in the phase to height conversion. Results have shown that at many ground control points (GCPs) the fused DEM is giving improved elevation values than Cartosat-1 DEM, which are near to DGPS elevation values by $0.5 \mathrm{~m}$ to $2 \mathrm{~m}$ as compared to Cartosat- 1 DEM.

\section{Conclusion}

The study highlighted that the quality of DEM generated using microwave InSAR pair improves with use of GCPs and a reference DEM. Results have shown that at many places the fused DEM is giving improved elevation values than Cartosat-1 DEM. ALOS PALSAR data being in LBand penetrates relatively more than the C-band (SRTM/Envisat ASAR) and also results in more accurate terrain elevation values at places of sparse vegetation and is comparable to DGPS values. In the present study the coherence in ALOS PALSAR was found better than the Envisat data. The large differences in elevation values at places are attributed to the hilly terrain, dense vegetation and coarse pixel size of data. A cognitively engineered algorithm with even finer resolution datasets can result in giving a better topographical representation using multisource, multi-sensor data particularly for inaccessible areas and extra-terrestrial bodies.

\section{Acknowledgement}

The authors are thankful to Dr. Y.V. N. Krishna Murthy, Director, Indian Institute of Remote Sensing, Dehradun for his encouragement and guidance. 


\section{References}

[1] Data Fusion Lexicon, US Department of Defence, 1991. Data fusion subpanel of the joint directors of laboratories. Technical Panel for C3, F.E. White, Code 4202. San Diego, CA: NOSC.

[2] Othman Sidek \& S.A. Quadri (2012): A review of data fusion models and systems, International Journal of Image and Data Fusion, 3:1, 3-21

[3] Jaime Esteban, Andrew Starr, Robert Willetts, Paul Hannah, Peter Bryanston-Cross (2005), A review of data fusion models and architectures: towards engineering guidelines, Neural Computing and Applications, 14 (4), 273-281.

[4] Stelios C. A. Thomopoulos "Sensor Integration And Data Fusion", Proc. SPIE 1198, Sensor fusion II: human and machine strategies, 6-9 November, Philadelphia, PA, 178191.

[5] Nelson, A., Reuter, H.I., Gessler, P., 2009. DEM production methods and sources. In: Hengl, T., Reuter, H.I. (Eds.), Geomorphometry: Concepts, Software, and Applications. Elsevier, Amsterdam, pp. 65-85.

[6] K. Gwinner, F. Scholten, F. Preusker, S. Elgner, T. Roatsch, M. Spiegel, R. Schmidt, J. Oberst, R. Jaumann, C. Heipke, Topography of Mars from global mapping by HRSC highresolution digital terrain models and orthoimages: Characteristics and performance, Earth and Planetary Science Letters 294 (2010) 506-519.

[7] P.V.Radhadevi, S.S.Solanki, V.Nagasubramanian, D.SudheerReddy, T.KrishnaSumanth, J. Saibaba, GeetaVaradan,An algorithm for geometric correction of full pass TMC imagery of Chandrayaan-1, Planetary and Space Science 79-80 (2013) 45-51

[8] Shridhar D. Jawak, Alvarinho J. Luis, Synergistic use of multitemporal RAMP, ICESat and GPS to construct an accurate DEM of the Larsemann Hills region, Antarctica, Advances in Space Research 50 (2012) 457-470.

[9] Grodecki, J and G. Dial (2001). IKONOS geometric accuracy. Proceedings of Joint Workshop of ISPRS Working Groups I/2, I/5 and IV/7 on High Resolution Mapping from Space 2001, University of Hanover, Hanover, Germany, Sept 19-21.

[10] Jacobsen K., 2006: ISPRS-ISRO Cartosat-1 Scientific Assessment Programme (C-SAP) Technical report - test areas Mausanne and Warsaw, ISPRS Com IV, Goa 2006, IAPRS Vol. 36 Part 4, pp. 1052-1056.
[11] Krishna Muthry, Y.V.N., Srinivasa Rao, S., Prakasa Rao, D.S., Jayaraman V. Analysis of DEM generated using Cartosat-I stereo data over Mausanne les Alphilles Cartosat scientific appraisal programme (CSAP TS-5). ISPRS 2008, 37, 1343- 1348.

[12] Krishnaswamy, M. and S. Kalyanaraman (2004). "Indian Remote Sensing Satellite Cartosat-1: Technical features and data products".

[13] D. Giribabu, S. Srinivasa Rao, Y.V.N. Krishna Murthy, Improving Cartosat-1 DEM accuracy using synthetic stereo pair and triplet, ISPRS Journal of Photogrammetry and Remote Sensing 77 (2013) 31-43.

[14] D. Massonnet, and K.L.Fiegl, Radar interferometry and its application to changes in the Earth's surface, Reviews of Geophysics 36(4) (1998), pp. 441-550.

[15] Iain H. Woodhouse, 2009, Introduction to Microwave Remote Sensing, Taylors \& Francis, Florida

[16] Y. Gorokhovich and A. Voustianiouk (2006). Accuracy assessment of the processed SRTM-based elevation data by CGIAR using field data from USA and Thailand and its relation to the terrain characteristics, Remote Sensing of Environment, Volume 104, Issue 4, 30 October 2006, Pages 409-415.

[17] Goncalves, J. A. and Oliveira, A. M. (2004) Accuracy analysis of DEM's derived from ASTER imagery. ISPRS XX Commission III WG III/2 — Science Faculty University of Porto, Portugal.

[18] Michael Gilichinsky, Dmitry Melnikov, Ivan Melekestsev, Natasha Zaretskaya, and Moshe Inbar, Morphometric measurements of cinder cones from digital elevation models of Tolbachik, volcanic field, central Kamchatka, Can. J. Remote Sensing, Vol. 36, No. 4, pp. 287-300, 2010.

[19] Ashutosh Bhardwaj, Kamal Jain, R.S. Chatterjee, Shefali Agrawal, "Comparative analysis of DEM generated from Lband ALOS PALSAR and Cartosat-1 stereo data: A case study of Dehradun and surroundings", Regional Conference on Geomatics for Good Governance, ISG, University of Kashmir, Sept. 13-14, 2011.

[20] Shaoping Deng, Jixian Zhang, Pingxiang Li, Guoman Huang, DEM Fusion and its Application in Mapping Topography of Complex Area, International Symposium on Image and Data Fusion (ISIDF), Aug. 2011, IEEE International. 